\title{
Traitement précoce des malocclusions de classe III : les faits
}

\author{
Philippe AMAT $^{*}$ \\ 19 place des Comtes du Maine, 72000 Le Mans, France
}

MOTS CLÉS :

Malocclusion de classe III /

Traitement orthopédique /

Traitement précoce /

Masque facial /

Revue systématique

KEYWORDS:

Class III malocclusion /

Orthopedic treatment /

Early treatment /

Facemask /

Systematic review
RÉSUMÉ - L'objectif de cet article est d'exposer les faits probants publiés pour répondre aux questions suivantes : 1) Le traitement précoce des malocclusions de classe III est-il efficace? 2) Quel dispositif thérapeutique est le plus efficace ? 3) Les résultats sont-ils stables? Les convictions de Jean Delaire et de l'auteur quant à l'utilité d'une prise en charge habituellement précoce de ces dysmorphies seront exposées dans un second article [4].

ABSTRACT - Early treatment for class III malocclusions: the facts. The goal of this article is to present some evidence based facts in order to answer the following questions: 1) Is early treatment of class III malocclusions effective? 2) Which therapeutic device is the most effective? 3) Are the results lasting? The positions of Jean Delaire and the author regarding the usefulness of routine early treatment for these dysmorphia will be discussed in a second article [4].

\section{Introduction}

Le traitement des malocclusions de classe III est réputé délicat, principalement en raison de leur schéma de croissance difficilement prévisible et potentiellement défavorable. Leur prise en charge soulève de nombreuses interrogations, dont celle du calendrier thérapeutique: quand faut-il traiter précocement et dans quelles circonstances estil préférable de temporiser et d'attendre la fin de la croissance pour recourir à une chirurgie orthognatique?

Lobjectif de cet article est de présenter les faits probants publiés pour répondre aux questions:

- Le traitement précoce des malocclusions de classe III est-il efficace?

- Quel dispositif thérapeutique est le plus efficace?

- Les résultats sont-ils stables?

Force est de constater que les données publiées sont loin d'être toutes aussi valides et incontestables qu'on pourrait idéalement l'espérer. Seul un nombre

\footnotetext{
* Auteur pour correspondance : amatph@noos.fr
}

restreint de nos traitements repose sur des données incontestables et il nous faut gérer au mieux l'incertitude. Parfois, nous n'avons d'autre choix que d'utiliser, avec réserve et prudence, quelques rares études de qualité méthodologique inégale, notre expérience et/ou celle de nos confrères comme seules sources d'informations. C'est le vaste champ des convictions qui sont scientifiquement licites, dès lors qu'elles sont exposées en tant que telles. Un second article [4] présentera les concepts thérapeutiques qui sous-tendent ces convictions et leur mise en œuvre pour trois cas cliniques.

\section{Rappels sur le syndrome de classe III}

La définition proposée par Angle en 1899 [7], qui décrit la classe III par la protrusion de la mandibule et de l'arcade dentaire telle qu'observée au niveau des premières molaires, n'est qu'un symptôme commun aux classes III. Elle ne fournit pas d'indications diagnostiques ou thérapeutiques.

Les classes III peuvent être définies comme un syndrome [62], c'est-à-dire un ensemble de symptômes ou de signes constituant une individualité 
clinique, mais non étiologique. Les dysmorphies de classe III présentent une grande variété anatomique et étiopathogénique $[13,18,20,23,27,34$, $44,46]$ reflet d'une interaction entre facteurs héréditaires [40] et environnementaux [48] (ventilation orale, dysfonctions linguales, parafonctions, etc.). Elles associent fréquemment à l'anomalie sagittale des anomalies des dimensions transversale et verticale, et elles peuvent n'être qu'un des éléments d'un syndrome dysmorphique cranio-facial (trisomie 21, achondroplasie, etc.).

La prévalence des malocclusions de classe III varie en fonction de l'appartenance ethnique de la population étudiée. Elle a été estimée de 1 à $5 \%$ des sujets de type caucasien $[25,28,34,56,59]$. La prévalence est plus importante dans les populations de type asiatique, de l'ordre de 9 à $19 \%$ [11, 15,33].

Les syndromes de classe III peuvent affecter tous les constituants squelettiques, alvéolaires et dentaires. L'organisme s'adaptant à la dysmorphie, les formes cliniques sont généralement mixtes et les constituants impliqués (typologie crânienne, dimension des maxillaires et leurs rapports conjoints ou avec la base crânienne) peuvent s'amplifier ou se compenser.

Outre un examen clinique précis, dont un bilan de l'ensemble des fonctions orofaciales, leur diagnostic requiert une étude complète des caractéristiques cranio-cervico-faciales de la dysmorphie, préférentiellement au moyen de l'analyse architecturale et structurale de Delaire [19].

La pluralité des formes cliniques du syndrome de classe III impose le recours à des traitements spécifiques. Le choix thérapeutique repose principalement sur un diagnostic étiologique précis et la connaissance des indications et limites des diverses approches thérapeutiques.

Plus de $50 \%$ des dysmorphies de classe III présentent une "insuffisance maxillaire » $[13,20,27]$ dont le traitement par traction à direction postéroantérieure sur masque de Delaire est de meilleur pronostic que celui du traitement d'une prognathie mandibulaire vraie [61].

\section{Les faits probants publiés}

\subsection{La démarche factuelle}

Lorthodontie fondée sur les faits [3] (OFF) désigne l'utilisation des principes de l'approche factuelle $[51,55]$ en orthodontie. C'est un outil pour rechercher rapidement les informations utiles aux décisions cliniques. Il permet d'en évaluer la qualité méthodologique et de sélectionner les meilleures d'entre elles. Enfin, en les associant à notre compétence clinique individuelle, il aide à délivrer les soins les plus efficaces, en tenant compte des besoins et de la situation de nos patients [2].

Pour être utiles à la résolution d'un problème clinique spécifique, les données publiées doivent associer validité scientifique (degré d'exactitude évalué avec la hiérarchie des protocoles de recherche) et pertinence clinique (utilité et applicabilité à notre pratique clinique).

Afin d'éviter au clinicien de réaliser lui-même l'évaluation critique d'un grand nombre de données, des sources d'informations fournissent des données probantes qui ont été préévaluées. Elles appliquent un processus d'évaluation critique, afin de ne retenir que les études de la plus haute qualité. Elles sont régulièrement réactualisées.

\subsection{Pyramide des « $6 S$ »}

Afin de faciliter leur recherche, Haynes [21] a classé les meilleures sources de données préévaluées, en une hiérarchie à six niveaux : la pyramide des « $6 S$ » (Fig. 1).

Chaque niveau de la pyramide reposant sur les données issues des niveaux inférieurs, la qualité scientifique et le degré de synthèse des données vont décroissant de haut en bas. La recherche d'informations doit donc débuter par la consultation des données situées au plus haut niveau possible de la pyramide. Inversement, le nombre et la gratuité d'accès de ces données vont croissants, de haut en bas de la pyramide des $« 6 S »$.

\subsection{Utilisation de la pyramide des « $6 S$ »}

La description des méthodes d'interrogation des sites sort du cadre de cet article. Elles sont exposées par ailleurs [2].

\subsubsection{Les systèmes}

Ce sont des systèmes informatisés d'aide à la prise de décisions cliniques qui ne sont pas encore disponibles en odontologie. On sautera donc systématiquement ce niveau de la pyramide des « $6 S$ ». 


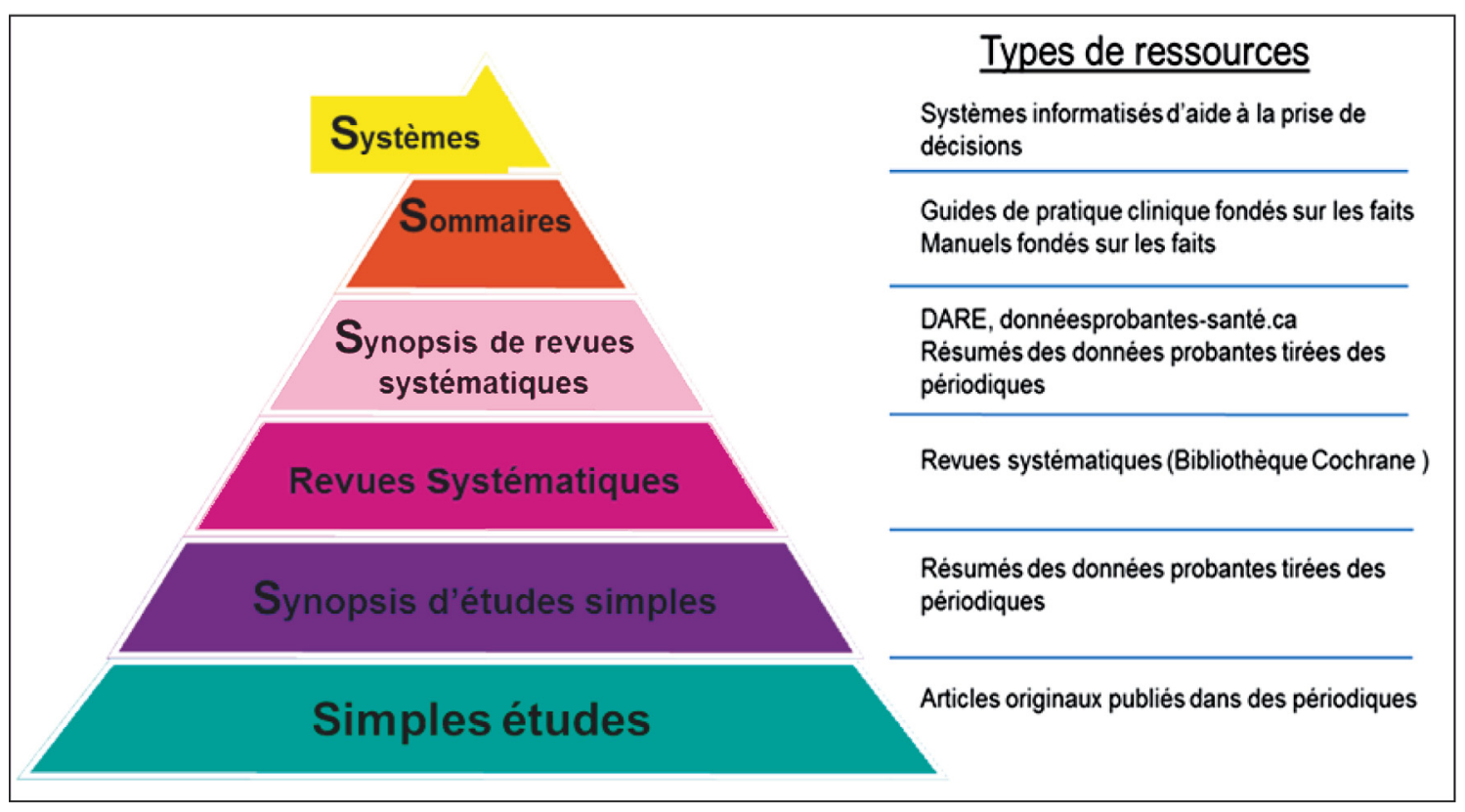

Figure 1

Pyramide des « $6 S$ » des sources de données probantes pré-évaluées et issues de la recherche (adapté de DiCenso, Bayley et Haynes, 2009 [21]).

\subsubsection{Les sommaires}

Les sommaires intègrent les meilleures données disponibles à des niveaux inférieurs de la pyramide des « $6 \mathrm{~S}$ ». Ils sont régulièrement réactualisés et fournissent l'ensemble des meilleures approches thérapeutiques, pour répondre à une question clinique donnée. Ils apportent une réponse globale au problème clinique, à la différence des niveaux d'informations inférieurs de la pyramide, qui n'abordent le plus souvent qu'un seul aspect d'une question.

Ce sont principalement les guides de pratique clinique fondés sur les faits (GPCFF).

On trouve principalement ces GPCFF sur les sites de la Haute Autorité de Santé (HAS) et de la National Guidelines Clearinghouse.

\subsubsection{Site de la Haute autorité de santé (www.has.sante.fr)}

Son interrogation avec le terme MeSH [29] « Malocclusion de classe III » fournit quatre GPCFF (recommandation de bonne pratique), dont un seul est en rapport avec la question posée :

- Indications de l'orthopédie dento-faciale et dento-maxillo-faciale chez l'enfant et l'adolescent [31].
La HAS recommande :

« L'âge optimal du traitement ne peut être fixé uniquement en fonction de l'anomalie. D'autres facteurs doivent être pris en compte pour le déterminer, tels que l'état général, les conditions psychiques et sociales, l'âge dentaire, le stade de croissance et de maturation, les anomalies associées. »

"Pour le cas spécifique des malocclusions de classe III, les éléments cités ci-dessus étant supposés favorables", par consensus, le groupe de travail de la HAS recommande "de traiter le plus tôt possible les proglissements mandibulaires et de différer le traitement des classes III d'origine purement dentaire jusqu'à, au plus tôt, la denture adolescente stable. »

«Le traitement des classes III squelettiques d'origine fonctionnelle doit être mis en œuvre le plus précocement possible (rééducation des fonctions orofaciales et avancée maxillaire en utilisant le masque facial de Delaire).

Le traitement des classes III squelettiques d'origine héréditaire distingue:

- le traitement de la brachygnathie maxillaire, avec expansion maxillaire mais toujours avec une avancée maxillaire. Il se ramène au traitement orthopédique des classes III d'origine fonctionnelle, la disjonction intermaxillaire préalable dicte la date de début de traitement; 
- le traitement du prognathisme mandibulaire, pour lequel la chirurgie orthognathique en denture adulte en fin de croissance est préférée à l'action frénatrice de la fronde occipito-mentonnière. »

\subsubsection{Site de la National Guidelines Clearinghouse (www.guidelines.gov)}

Son interrogation au moyen du terme MeSH anglais [29] «Malocclusion, Angle Class III » fournit trois GPCFF, dont un seul est en rapport avec la question posée :

- Recommandation pour la prise en charge de la dentition et de l'occlusion en odontologie pédiatrique [6].

Il offre quelques éléments de réponse :

«Le traitement des malocclusions de classe III est indiqué :

- pour fournir des bénéfices psychosociaux à l'enfant en réduisant ou en supprimant la défiguration faciale;

- pour réduire la sévérité de la malocclusion en favorisant une croissance harmonieuse.

Le traitement précoce des classes III a été proposé depuis plusieurs années et préconisé en tant qu'outil nécessaire à la pratique d'une orthopédie dento-faciale contemporaine. Il peut fournir un environnement plus favorable à la croissance et à l'amélioration de l'occlusion, des fonctions et de l'esthétique. Bien qu'un traitement précoce puisse réduire la malocclusion et potentiellement supprimer le besoin d'une chirurgie orthognathique ultérieure, cela n'est pas toujours possible.

En général, la croissance des patients présentant une classe III se poursuit plus tardivement et de façon moins prévisible. En conséquence, un traitement associant orthodontie et chirurgie peut être la meilleure option pour conduire certains patients vers un résultat satisfaisant.

Le traitement interceptif d'une malocclusion de classe III devrait aboutir à une amélioration du recouvrement, du surplomb, de l'engrènement des dents postérieures et à un aspect esthétique et un profil compatibles avec les caractéristiques squelettiques du patient. »

\subsubsection{Les synopsis de revues systématiques}

Un synopsis de revue systématique est un résumé succinct d'une revue systématique, sélectionnée pour sa grande qualité méthodologique. Sa valeur ajoutée par rapport à la revue systématique est l'évaluation critique, par des experts, de la qualité méthodologique et de l'applicabilité clinique des résultats de cette revue.

On consulte en accès libre la base des résumés des revues sur les effets ou Database of Abstracts of Reviews of Effects (DARE) [30]. Son interrogation au moyen du terme MeSH « Malocclusion, Angle Class III » fournit trois références de revues systématiques, qui n'ont pas encore bénéficié d'une évaluation par des experts et ne sont pas présentées sous forme de synopsis à ce jour. Deux d'entre elles sont en rapport avec deux de nos questions.

\subsubsection{Le traitement précoce des malocclusions de classe III est-il efficace?}

Une revue systématique [57] a été consacrée aux résultats du traitement orthopédique des malocclusions de classe III. Le niveau de qualité des études sélectionnées variait de faible (quatre études) à moyen/élevé (cinq études). Les données des études de niveau de qualité méthodologique moyen/élevé décrivaient un taux de succès des traitements orthopédiques de malocclusions de classe III (traitement par disjonction maxillaire et masque facial) de plus de $76 \%$ évalué cinq ans après la fin du traitement orthopédique.

\subsubsection{Quel dispositif thérapeutique est le plus efficace?}

Les auteurs d'une revue systématique [41] concluent à une insuffisance des données pour pouvoir proposer des recommandations claires concernant l'efficacité d'un traitement par fronde mentonnière pour retarder la croissance mandibulaire.

\subsubsection{Les revues systématiques}

Une revue systématique est une revue de la littérature, pour laquelle l'exhaustivité des études publiées ou non et qui traitent d'une question clinique précise, est recherchée, évaluée de manière critique et synthétisée. Parfois, ces études peuvent être pertinentes et contradictoires. La conduite d'une revue doit tout particulièrement limiter l'introduction d'erreurs aléatoires et systématiques (ou biais) [51].

On consulte la base des revues systématiques Cochrane et Medline. 


\subsubsection{La base des revues systématiques Cochrane: Cochrane Database of Systematic Reviews (CDSR, http://onlinelibrary.wiley.com/ book/10.1002/14651858/titles)}

Elle recense les revues systématiques Cochrane et les protocoles des revues, annoncées et en cours. L'interrogation au moyen du terme MeSH « Malocclusion, Angle Class III » n'identifie aucune revue systématique Cochrane répondant à nos questions.

\subsubsection{La base de données Medline (accès gratuit par PubMed, www.pubmed.gov)}

Elle constitue la source d'information la plus complète et la plus facilement accessible sur les soins de santé. Il existe trois modes de recherche usuels sur PubMed [2] : recherche en langage naturel, recherche avancée et recherche avec le MeSH Database.

On choisit le mode recherche simple avec le terme MeSH «Malocclusion, Angle Class III » et on accède aux seules revues systématiques de la littérature en cliquant sur le filtre systematic reviews (revues systématiques).

Parmi les 21 références trouvées, 8 revues de la littérature $[5,14,22,26,35,37,41,57]$ abordent les questions objets de cet article. Trois d'entre elles [5,41,57] ont déjà été identifiées à des niveaux supérieurs de la pyramide des « $6 \mathrm{~S}$ ».

Les conclusions des cinq revues de la littérature restantes peuvent être regroupées pour répondre à nos questions.

\subsection{Le traitement précoce des malocclusions de classe III est-il efficace?}

Selon une méta-analyse [37] consacrée à l'efficacité du traitement par masque facial de protraction, les traitements par protraction sont efficaces. Langle ANB s'accroît de $0,9^{\circ}$ à $4,39^{\circ}$ suivant l'étude, avec une valeur moyenne de $2,79^{\circ}$, bien supérieure à l'effet de la croissance annuelle.

La même méta-analyse [37] a évalué l'influence de l'âge des patients sur les résultats du traitement. Les études sélectionnées ont été réparties dans un groupe d'âge 4-10 ans et un groupe d'âge 10-15 ans. Les données indiquent que le traitement par masque facial de protraction est efficace chez des patients en croissance, mais à un moindre degré après 10 ans.
Le traitement par protraction maxillaire est efficace [35]. Ses effets sont squelettiques, mais aussi dento-alvéolaires au niveau maxillaire et mandibulaire. Toutefois, les effets ont été étudiés immédiatement après la fin du traitement orthopédique, sans suivi à long terme.

Une réunion de consensus de l'Association des orthodontistes néo-zélandais [22] a conclut que «les possibilités d'action des dispositifs sur la croissance sont très limitées, les effets orthopédiques à court terme pourraient jouer un rôle dans la correction des malocclusions et une grande variabilité des réponses a été observée dans les études».

\subsection{Quel dispositif thérapentique est le plus efficace?}

Selon une méta-analyse [37] consacrée à l'efficacité du traitement par masque facial de protraction, les seules différences observées entre les patients traités par protraction seule et ceux traités par protraction associée à une disjonction maxillaire rapide concernent l'inclinaison de l'incisive maxillaire plus marquée chez les patients traités sans expansion, et la durée de traitement, qui est plus brève quand une disjonction est associée.

\subsection{Les résultats sont-ils stables?}

Une étude [63], sélectionnée dans une récente revue de la littérature [14], montre que le traitement par protraction avec masque facial associée à une disjonction maxillaire rapide peut corriger à long terme (suivi de 5 ans 1/2) les malocclusions de classe III chez des enfants d'un âge moyen de 8 ans et 3 mois. Néanmoins, les auteurs de la revue concluent que les données publiées sont insuffisantes pour tirer des conclusions sur la stabilité à long terme du traitement des classes III et la satisfaction des patients.

Une revue [26], consacrée à la prédiction des résultats de traitement des malocclusions de classe III, a permis de sélectionner 14 études et d'identifier 38 indicateurs différents de résultat de traitement : 35 céphalométriques et trois issus de l'analyse des moulages d'étude. Des modèles prévisionnels comportant trois à quatre indicateurs de prévision ont été répertoriés dans la plupart des études.

Le niveau de qualité méthodologique des études varie de faible à moyen. En raison de la grande diversité des indicateurs et des différences entre les modèles prévisionnels proposés, l'existence d'un 


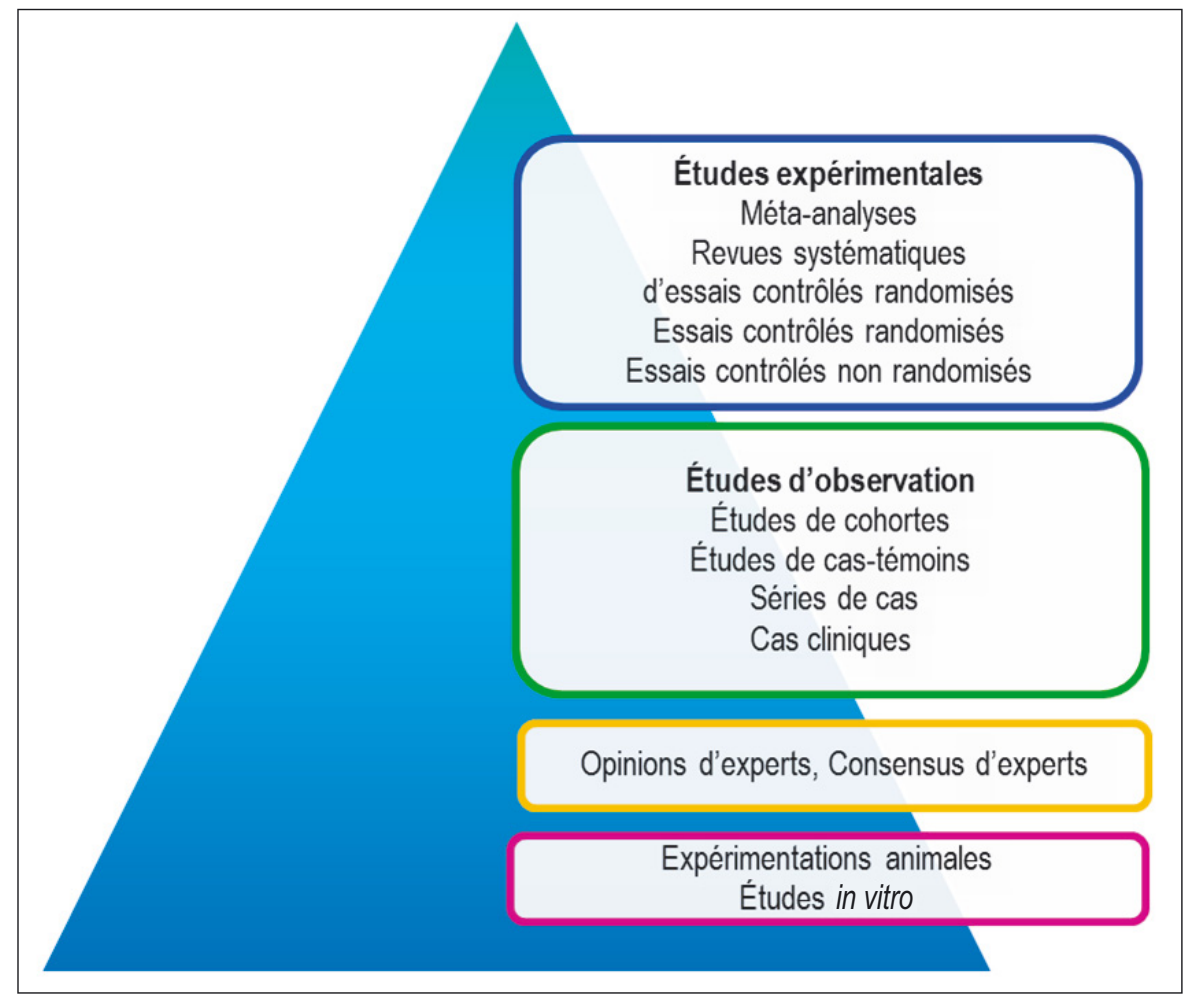

Figure 2

Pyramide de la hiérarchie des faits pour une question de traitement [24, 45, 49].

indicateur universel du résultat de traitement des malocclusions de classe III peut être mise en doute.

\subsubsection{Les synopsis d'études simples}

Un synopsis d'étude simple est un résumé succinct d'une étude simple, sélectionnée pour sa grande qualité méthodologique. Le texte tient généralement en une page et fournit toute, et rien que, l'information nécessaire et suffisante pour répondre à la question clinique. Des commentaires sont ajoutés par des experts qui évaluent la qualité méthodologique de l'étude simple et l'applicabilité clinique de ses résultats.

On peut les trouver dans des journaux fondés sur les faits comme Evidence-Based Dentistry (http:// www.nature.com/ebd/index.html). La consultation de ce journal offre un synopsis d'étude simple en rapport avec deux de nos interrogations. C'est un essai clinique randomisé prospectif [60] étudiant les effets du traitement par protraction maxillaire avec ou sans disjonction maxillaire rapide. Il fait partie des études sélectionnées dans la revue systématique de Toffol, et al. [57], classée au niveau des synopsis de revues systématiques.

\subsubsection{Le traitement précoce des malocclusions de classe III est-il efficace?}

Les auteurs du même essai clinique randomisé [60] concluent que le traitement par masque facial de protraction permet une correction efficace chez les jeunes patients avec une malocclusion de classe III.

\subsubsection{Quel dispositif thérapentique est le plus efficace?}

Les auteurs du même essai clinique randomisé [60] concluent que l'utilité d'une disjonction maxillaire rapide en l'absence de déficit transversal ou d'occlusion croisée d'étiologie squelettique ou dento-alvéolaire, n'est pas avérée au regard des résultats de cette étude.

\subsubsection{Les études simples}

Ces études simples n'ont pas été pré-évaluées. Leur évaluation critique [2] est facilitée par l'évaluation de leur protocole de recherche à l'aide de la hiérarchie des faits pour une question de traitement $[24,45,49]$ (Fig. 2) et des grilles de lecture 
Tableau 1

Critères d'inclusion et d'exclusion des études.

\begin{tabular}{|l|l|}
\hline \multicolumn{1}{|c|}{ Critères d'inclusions } & \multicolumn{1}{c|}{ Critères d'exclusions } \\
\hline Méta-analyses, essais cliniques randomisés et & Cas cliniques, séries de cas, études descriptives, \\
essais cliniques contrôlés & revues de la littérature, articles d'opinion, résumés \\
Études écrites en anglais ou en français & Études en laboratoire \\
Études publiées entre janvier 2006 et dé- & Études menées chez des adultes \\
cembre 2012 & Études sur l'association entre malocclusions de \\
Études portant sur des patients en croissance & classe III et malformations cranio-faciales \\
Études menées sur des téléradiographies de & Études de prévalence des malocclusions de \\
profil et mentionnant les mesures des lon- & classe III \\
gueurs totales maxillaire et mandibulaire, et & Études de prédiction de croissance \\
des relations intermaxillaires dans les dimen- & Études comparant différentes malocclusions \\
sions sagittale et verticale & Études traitant de l'association entre malocclusion \\
Groupe de témoins en classes III non traités & de classe III et dysfonctionnements de l'appareil \\
& manducateur \\
& Études sans groupe de témoins non traités ou sans \\
& groupe de contrôle normal \\
& Études sur moulages d'études ou sans analyses cé- \\
& phalométriques \\
& Traitements avec extractions \\
& Traitements associés à une chirurgie orthogna- \\
thique & Cas sélectionnés parmi les succès thérapeutiques \\
\hline
\end{tabular}

élaborées par le groupe de médecine fondée sur les faits de l'Université McMaster [50, 52].

La plus récente revue systématique répondant à nos questions : Résultats du traitement orthopédique des malocclusions de classe III : une revue systématique [57], a été publiée en 2008 et portait sur les études publiées entre janvier 1966 et décembre 2005.

Nous allons chercher les simples études publiées depuis cette date.

\subsubsection{Stratégie de recherche}

Il n'entre pas dans l'objectif de cet article de mener une revue systématique de la littérature. Le but est d'actualiser les données de la dernière revue systématique consacrée aux traitements précoces de classe III [57] en identifiant les études publiées depuis la réalisation de cette revue et qui pourraient nous aider à répondre aux trois questions, objectif de cet article.

La recherche bibliographique, semblable à celle de Toffol, et al. [57], a donc porté sur les études publiées entre janvier 2006 et décembre 2012.

La base de données Medline a été interrogée avec le terme MeSH «Malocclusion, Angle Class III » que l'on a croisé avec les termes MeSH « orthodontics, interceptive » et «orthodontics, corrective».

Cette recherche documentaire a été complétée par une interrogation de la base Registre central Cochrane des essais cliniques contrôlés (CENTRAL: Cochrane central register of controlled trials).

\subsubsection{Critères de sélection des études}

Les critères d'inclusion et d'exclusions des études utilisés sont regroupés dans un tableau (Tab. 1). Ont été sélectionnés les types d'études suivants : métaanalyses, essais cliniques randomisés et essais cliniques contrôlés.

Les études sélectionnées doivent recourir à des téléradiographies de profil pour analyser les effets $\mathrm{du}$ traitement orthopédique sur les longueurs totales maxillaire et mandibulaire, et les relations intermaxillaires dans les dimensions sagittale et verticale. Les études doivent comporter un groupe de témoins en classe III non traités et aucune restriction n'a été fixée pour la taille de l'échantillon.

\subsubsection{Collecte des données et analyse}

Sur la page Advanced search (recherche avancée) de PubMed, l'aide à l'élaboration d'une stratégie de 
recherche (Search builder) permet de croiser plusieurs critères de recherche de façon guidée [2]. Après avoir sélectionné un champ de recherche, on tape un terme dans la fenêtre d'interrogation, puis on choisit l'opérateur logique désiré (and, or, not) dans le menu déroulant placé à gauche de la barre d'interrogation.

Les opérateurs logiques permettent de combiner entre eux deux ou plusieurs termes d'interrogation, afin d'élargir ou de restreindre la recherche bibliographique et de constituer l'équation de recherche, utilisée par PubMed pour traduire cette recherche.

Nous avons utilisé l'équation de recherche: \#10: Search ((\#9) OR \#7) OR \#5 avec:

\#1 : Search malocclusion, Angle Class III

\#2 : Search ("2006/01/01"[Date - Publication] : "2012/12/31"[Date - Publication])

\#3 : Search (\#2) AND \#1

\#4 : Search "controlled clinical trial"[Publication Type]

\#5: Search (\#3) AND \#4

\#6 : Search "randomized controlled trial"[Publication Type]

\#7 : Search (\#6) AND \#3

\#8 : Search "meta analysis"[Publication Type]

\#9 : Search (\#8) AND \#3

Linterrogation de Medline a été complétée par celle du Registre central Cochrane des essais cliniques contrôlés.

\subsubsection{Résultats}

La stratégie de recherche a permis d'identifier 41 études. Après application des critères d'inclusion et d'exclusion recensés dans le tableau 1, 18 études ont été retenues. Six d'entre elles ont été conduites en Turquie $[8,9,32,36,38,58]$, trois en Italie $[10,16$, 43], deux au Royaume-Uni [42,53], deux aux ÉtatsUnis [12, 17], une en Iran [54], une en Chine [41], une à Taiwan [39], une en Colombie [47] et une en Égypte [1].

Les motifs principaux d'exclusion étaient : traitements associés à une chirurgie orthognathique, études menées chez des adultes, études comparative de différents brackets, études traitant de l'association entre malocclusion de classe III et dysfonctionnements de l'appareil manducateur et études sur l'association entre malocclusions de classe III et malformations cranio-faciales.
Linterrogation du Registre central Cochrane des essais cliniques contrôlés n'a pas permis d'identifier d'autres études.

\subsubsection{Types d'étude et modalités de traitement}

Les 18 études comprennent une métaanalyse [41], trois essais cliniques randomisés $[38,42,54]$ et quatorze essais cliniques contrôlés $[1,8-10,12,16,17,32,36,39,43,47,53,58]$.

Les articles étudient les effets du traitement par masque facial [42], par masque facial associé à un plan de morsure [16] ou à une disjonction maxillaire rapide [43], ou par masque facial associé à une disjonction maxillaire rapide et suivi d'un traitement multi-bracket [8].

Sont également étudiés les effets du traitement par masque facial associé ou non à une disjonction maxillaire rapide [58], les effets comparés du traitement par masque facial associé à une disjonction rapide maxillaire simple ou à une disjonction rapide maxillaire avec séquences d'activationdésactivation [32].

D'autres études décrivent les effets comparés de la fronde mentonnière et du masque facial [54], ceux du Reverse Twin-Block (pour classes III) et du masque facial [53], les effets du traitement par fronde mentonnière [40], par fronde mentonnière avec forces légères [12], ou par fronde mentonnière avec deux intensités différentes de forces [1].

Une étude est consacrée aux effets des traitements par masque facial ou par Jasper Jumper modifié pour les classes III, sur les dysfonctionnements de l'appareil manducateur [38].

D'autres publications étudient les effets du traitement par élastiques intermaxillaires de classe III tendus entre des mini-plaques d'ancrage squelettique [17], par régulateur fonctionnel (FR-3) de Fränkel [36], par le dispositif orthopédique OMA [39], par un système de protraction bimaxillaire modifié (modified TTBA) [9] et par une traction cervicale appliquée sur les premières molaires mandibulaires $[10,47]$.

\subsubsection{Conclusions des études}

Rappelons qu'il n'entre pas dans l'objectif de cet article de mener une revue systématique de la littérature, mais de rapporter les conclusions des essais cliniques sélectionnés parmi les publications postérieures à la revue systématique de Toffol, et al. [57]. 
Lanalyse quantitative des données actuellement publiées est pratiquement irréalisable en raison de la grande hétérogénéité des mesures céphalométriques retenues, des calendriers et des durées de traitement, ainsi que de l'âge des sujets étudiés.

On peut néanmoins regrouper les éléments de réponse de ces études à nos trois interrogations.

\subsection{Le traitement précoce des malocclusions de classe III est-il efficace?}

Si l'indication en est pertinente, le traitement par masque facial associé ou non à une disjonction maxillaire rapide [58] peut être un traitement efficace des classes III.

Le traitement orthopédique précoce des classes III par masque de protraction facial chez des patients de moins de 10 ans [42] est efficace à court terme au niveau squelettique et dento-alvéolaire. Il n'induit pas de dysfonctionnements de l'appareil manducateur. Un résultat clinique favorable, défini par l'obtention d'un surplomb positif, est obtenu pour $70 \%$ des patients. Toutefois, un traitement précoce ne semble pas apporter un bénéfice psychosocial cliniquement significatif.

Les effets d'un traitement par masque facial associé à une disjonction maxillaire rapide et suivi d'un traitement multi-bracket [8] sont principalement observés au niveau de la mandibule et des structures dento-alvéolaires.

Une déficience maxillaire peut efficacement être corrigée par un traitement avec fronde mentonnière ou masque facial [54].

Les données sont insuffisantes pour pouvoir donner des recommandations claires concernant l'efficacité à court terme d'un traitement par fronde mentonnière pour retarder la croissance mandibulaire [41].

Le traitement par fronde mentonnière [1] améliore les relations maxillo-mandibulaires chez les patients en classe III et en croissance, mais l'amélioration squelettique est faible.

Moins de $50 \%$ des traitements réalisés par fronde mentonnière avec des forces légères [12] mènent à un résultat clinique favorable. La correction de la malocclusion de classe III résulte de modifications dento-alvéolaires significatives et sans modifications orthopédiques au niveau de la mandibule. En association avec le port de la fronde mentonnière, une expansion maxillaire par quad-hélix pourrait aider à la correction de la malocclusion de classe III.

Les rapports d'occlusion de classe III peuvent être efficacement améliorés par un traitement avec Reverse Twin-Block (pour classes III) ou par masque facial [53].

Le traitement par régulateur fonctionnel (FR-3) de Fränkel [36] des patients en classe III avec un profil concave conduit à une amélioration significative au niveau des tissus mous et durs.

Le traitement par un système de protraction bimaxillaire modifié (modified TTBA) [9] appliqué à deux groupes d'âge squelettique différent $(8,18 \pm$ $0,50$ et $11,75 \pm 1,00)$ permet une correction dentaire et squelettique des malocclusions de classe III.

Le traitement par un dispositif orthopédique OMA [39] permet une correction efficace des malocclusions de classe III avec déficience maxillaire et prognathisme mandibulaire chez des enfants en croissance.

Le traitement de sujets en classe III, avant la poussée de croissance pubertaire (âge moyen, $11,10 \pm 1,8)$, par élastiques intermaxillaires de classe III tendus entre des mini-plaques d'ancrage squelettique [17] induit une avancée moyenne de $4 \mathrm{~mm}$ des structures squelettiques et des tissus mous maxillaires, ainsi qu'une amélioration supérieure à $2 \mathrm{~mm}$ au niveau de la mandibule (l'évaluation a été menée un an après traitement).

\subsection{Quel dispositif thérapentique est le plus efficace?}

La comparaison des effets du traitement par masque facial associé à une disjonction rapide maxillaire simple ou à une disjonction rapide maxillaire avec séquences d'activation-désactivation [32] montre l'effet positif de cette seconde option sur la protraction maxillaire avec une avancée prononcée du point $\mathrm{A}$.

Les traitements par masque facial ou fronde mentonnière [54] sont tous les deux efficaces dans la correction d'une déficience maxillaire.

Le traitement par fronde mentonnière avec deux intensités différentes de forces [1] conduit à des effets similaires, à l'exception d'une réduction plus prononcée de la hauteur ramale avec les forces élevées.

La correction des malocclusions de classe III par un masque facial est à dominante squelettique, essentiellement par avancée maxillaire, et celle 
du Reverse Twin-Block est principalement dentoalvéolaire [53].

$\mathrm{Ni}$ le traitement par masque facial, ni celui par Jasper Jumper modifié pour les classes III, n'entraînent de dysfonctionnements de l'appareil manducateur [38].

Outre un résultat clinique favorable au niveau du maxillaire, l'association d'un plan de morsure mandibulaire au port d'un masque facial [16] permet un contrôle efficace de la rotation mandibulaire avec une fermeture progressive de l'angle goniaque.

\subsection{Les résultats sont-ils stables?}

À long terme, le traitement des patients en classe III par masque facial associé à une disjonction maxillaire [43] rapide conduit à $73 \%$ de succès thérapeutiques. Lévolution favorable des rapports squelettiques repose principalement sur l'amélioration significative de la position de la mandibule dans la dimension sagittale.

Les effets dento-squelettique favorables d'un traitement par traction cervicale appliquée sur les premières molaires mandibulaires suivi d'une phase par dispositif multi-attache $[10,47]$ restent stables à long terme. Les effets sont principalement une avancée et une augmentation de longueur mandibulaires de moindre importance. On observe également une rotation postérieure significative de la mandibule. Les patients en classe III qui n'ont pas été traités n'ont présenté aucune tendance à l'amélioration spontanée lors de l'observation post-pubertaire.

\section{Conclusion}

Force est de constater que les faits publiés sur les dysmorphies de classe III sont en nombre plus restreint que ceux consacrés aux classes II et que leur niveau de qualité méthodologique est variable.

Globalement, les données probantes publiées semblent montrer que le traitement précoce des malocclusions de classe III est efficace, particulièrement par masque facial associé ou non à une disjonction maxillaire, et que ses résultats sont stables.

Il serait néanmoins souhaitable qu'un essai clinique randomisé soit consacré aux effets des différentes modalités de traitement orthopédique avec une évaluation en fin de croissance cranio-faciale.

\section{Bibliographie}

[1] Abdelnaby YL, Nassar EA. Chin cup effects using two different force magnitudes in the management of Class III malocclusions. Angle Orthod 2010;80:957-962.

[2] Amat P. Dentisterie fondée sur les faits : en omnipratique et en orthodontie. Paris : Éditions CdP, 2012.

[3] Amat P. Orthopédie dento-faciale fondée sur les faits: marotte d'universitaire ou indispensable outil clinique quotidien ? Rev Orthop Dento Faciale 2006;40:421-451.

[4] Amat P, Delaire J. Traitement précoce des malocclusions de classe III : les convictions. Orthod Fr 2013;84:53-70.

[5] American Academy of Pediatric Dentistry. Clinical guideline on management of the developing dentition in pediatric dentistry. Pediatr Dent 2004;26(7 Suppl):128-131.

[6] American Academy of Pediatric Dentistry (AAPD). Guideline on management of the developing dentition and occlusion in pediatric dentistry. Chicago (IL): AAPD, 2009, $13 \mathrm{p}$.

[7] Angle EH. Classification of malocclusion. The Dental Cosmos 1899;41:248-264.

[8] Arman A, Ufuk Toygar T, Abuhijleh E. Evaluation of maxillary protraction and fixed appliance therapy in Class III patients. Eur J Orthod 2006;28:383-392.

[9] Atalay Z, Tortop T. Dentofacial effects of a modified tandem traction bow appliance. Eur J Orthod 2010;32:655-661.

[10] Baccetti T, Rey D, Oberti G, Stahl F, McNamara JA Jr. Longterm outcomes of Class III treatment with mandibular cervical headgear followed by fixed appliances. Angle Orthod 2009;79:828-834

[11] Baik HS, Han HK, Kim DJ, Proffit WR. Cephalometric characteristics of Korean Class III surgical patients and their relationship to plans for surgical treatment. Int J Adult Orthodon Orthognath Surg 2000;15:119-128.

[12] Barrett AA, Baccetti T, McNamara JA Jr. Treatment effects of the light-force chincup. Am J Orthod Dentofacial Orthop 2010;138:468-476.

[13] Battagel JM. The aetiological factors in Class III malocclusion. Eur J Orthod 1993;15:347-370.

[14] Bondemark L, Holm AK, Hansen K, et al. Long-term stability of orthodontic treatment and patient satisfaction. A systematic review. Angle Orthod 2007;77:181-191.

[15] Chan GK. Class III malocclusion in Chinese: etiology and treatment. Am J Orthod 1974;65:152-156.

[16] Cozza P, Baccetti T, Mucedero M, Pavoni C, Franchi L. Treatment and posttreatment effects of a facial mask combined with a bite-block appliance in Class III malocclusion. Am J Orthod Dentofacial Orthop 2010;138:300-310.

[17] De Clerck H, Cevidanes L, Baccetti T. Dentofacial effects of bone-anchored maxillary protraction: a controlled study of consecutively treated Class III patients. Am J Orthod Dentofacial Orthop 2010;138:577-581.

[18] Delaire J. L'emploi physiologique des tractions extraorales postéro-antérieures sur masque orthopédique dans le traitement des classes III. Orthod Fr 1988:59:577-589. 
[19] Delaire J. Lanalyse architecturale et structurale craniofaciale (de profil). Principes théoriques. Quelques exemples d'emploi en chirurgie maxillo-faciale. Rev Stomatol Chir Maxillofac 1978;79:1-33.

[20] Delaire J. Maxillary development revisited: relevance to the orthopaedic treatment of class III malocclusions. Eur J Orthod 1997;19:289-311.

[21] DiCenso A, Bayley L, Haynes B. Accessing pre-appraised evidence: fine tuning the $5 \mathrm{~S}$ model into a $6 \mathrm{~S}$ model. Evid Based Nurs 2009;12:99-101.

[22] Dysart PS. Education and research development group New Zealand association of orthodontists. Growth modification-report of an NZAO symposium. N Z Dent J 2005;101:17-18.

[23] Ellis E, McNamara Ja Jr. Components of adult Class III malocclusion. J Oral Maxillofac Surg 1984;42:295-305.

[24] Forrest JL. Introduction to the basics of evidence-based dentistry: 2. Concepts and skills. J Evid Base Dent Pract 2009:9:108-112

[25] Foster TD, Day AJ. A survey of malocclusion and the need for orthodontic treatment in a Shropshire school population. Br J Orthod 1974;1:73-78.

[26] Fudalej P, Dragan M, Wedrychowska-Szulc B. Prediction of the outcome of orthodontic treatment of Class III malocclusions - a systematic review. Eur J Orthod 2011;33:190-197.

[27] Guyer EC, Ellis EE III, McNamara JA Jr, Behrents RG. Components of Class III malocclusion in juveniles and adolescents. Angle Orthod 1986;56:7-30.

[28] Haynes S. The prevalence of malocclusion in English children aged 11-12 years. Rep Congr Eur Orthod Soc 1970:89-98.

[29] http://mesh.inserm.fr/mesh/index.htm.

[30] http://www.crd.york.ac.uk/crdweb/SearchPage.asp.

[31] http://www.has-sante.fr/portail/upload/docs/application/ pdf/orthodontie_rap.pdf.

[32] Isci D, Turk T, Elekdag-Turk S. Activation-deactivation rapid palatal expansion and reverse headgear in Class III cases. Eur J Orthod 2010;32:706-715.

[33] Ishii H, Morita S, Takeuchi Y, et al. Treatment effect of combined maxillary protraction and chincap appliance in severe skeletal Class III cases. Am J Orthod Dentofacial Orthop 1987;92:304-312

[34] Jacobson A, Evans WG, Preston CB, Sadowsky PL. Mandibular prognathism. Am J Orthod 1974;66: 140-171.

[35] Jäger A, Braumann B, Kim C, Wahner S. Skeletal and dental effects of maxillary protraction in patients with angle class III malocclusion. A meta-analysis. J Orofac Orthop 2001;62:275-284.

[36] Kilic N, Celikoglu M, Oktay H. Effects of the functional regulator III on profile changes in subjects with maxillary deficiency. Eur J Orthod 2010;32:729-734.

[37] Kim JH, Viana MA, Graber TM, Omerza FF, BeGole EA. The effectiveness of protraction face mask therapy: a meta-analysis. Am J Orthod Dentofacial Orthop 1999;115:675-685.
[38] Kurt H, Alioğlu C, Karayazgan B, Tuncer N, Kılıçoğlu H. The effects of two methods of Class III malocclusion treatment on temporomandibular disorders. Eur J Orthod 2011;33:636-641.

[39] Lin HC, Chang HP, Chang HF. Treatment effects of occipitomental anchorage appliance of maxillary protraction combined with chincup traction in children with Class III malocclusion. J Formos Med Assoc 2007;106:380-391.

[40] Litton SF, Ackermann LV, Isaacson RJ, et al. A genetic study of Class III malocclusion. Am J Orthod 1970;58:565-357.

[41] Liu ZP, Li CJ, Hu HK, Chen JW, Li F, Zou SJ. Efficacy of short-term chincup therapy for mandibular growth retardation in class III malocclusion. Angle Orthod 2011;81:162-168.

[42] Mandall N, DiBiase A, Littlewood S, et al. Is early Class III protraction facemask treatment effective? A multicentre, randomized, controlled trial: 15-month follow-up. J Orthod 2010;37:149-161.

[43] Masucci C, Franchi L, Defraia E, Mucedero M, Cozza P, Baccetti T. Stability of rapid maxillary expansion and facemask therapy: a long-term controlled study. Am J Orthod Dentofacial Orthop 2011;140:493-500.

[44] McNamara JA Jr, Brudon WL. Orthodontic and orthopedic treatment in the mixed dentition. Ann Arbor: Needham Press, 1993:118,119,126.

[45] NHS Centre for Reviews and Dissemination. Undertaking systematic reviews of research on effectiveness: CRD's guidance for carrying out or commissioning reviews. 2nd ed., York: NHS Centre for Reviews and Dissemination, University of York, 2001.

[46] Park JU, Baik SH. Classification of Angle Class III malocclusion and its treatment modalities. Int J Adult Orthodon Orthognath Surg 2001;16:19-29.

[47] Rey D, Angel D, Oberti G, Baccetti T. Treatment and posttreatment effects of mandibular cervical headgear followed by fixed appliances in Class III malocclusion. Am J Orthod Dentofacial Orthop 2008;133:371-378; quiz 476.el.

[48] Rokosi T, Schilli W. Class III anomalies: a coordinated approach to skeletal, dental, and soft tissue problems. J Oral Surg 1981;39:860-870.

[49] Sackett D. Rules of evidence and clinical recommendations. Can J Cardiol 1993:9:487-489.

[50] Sackett DL, Haynes RB, Guyatt GH, Tugwell P. Clinical epidemiology: a basic science for clinical medicine. 2nd ed., Boston: Little, Brown and Company, 1991.

[51] Sackett DL, Rosenberg WM, Gray JA, Haynes RB, Richardson WS. Evidence based medicine: what it is and what it isn't. BMJ 1996;312:71-72.

[52] Sackett DL, Strauss SE, Richardson WS, Rosenberg W, Haynes RB. Evidence-based medicine: how to practice and teach EBM. London: Churchill-Livingstone, 2000.

[53] Seehra J, Fleming PS, Mandall N, Dibiase AT. A comparison of two different techniques for early correction of Class III malocclusion. Angle Orthod 2012;82:96-101.

[54] Showkatbakhsh R, Jamilian A, Ghassemi M, Ghassemi A, Taban T, Imani $Z$. The effects of facemask and reverse chin cup on maxillary deficient patients. J Orthod 2012;39:95-101. 
[55] Straus SE, Richardson WS, Glasziou P, Haynes RB Evidence-based medicine: how to practice and teach EBM. 3rd ed., London: Churchill-Livingstone, 2005.

[56] Thilander B, Myrberg N. The prevalence of malocclusion in Swedish school children. Scand J Dent Res 1973;81:12-20.

[57] Toffol LD, Pavoni C, Baccetti T, Franchi L, Cozza P. Orthopedic treatment outcomes in Class III malocclusion: a systematic review. Angle Orthod 2008;78:561-573.

[58] Tortop T, Keykubat A, Yuksel S. Facemask therapy with and without expansion. Am J Orthod Dentofacial Orthop 2007;132:467-474.

[59] Tschill P, Bacon W, Sonko A. Malocclusion in the deciduous dentition of Caucasian children. Eur J Orthod 1997;19:361-367.
[60] Vaughn GA, Vaughn B, Moon HB, Turley PK. The effects of maxillary protraction therapy with or without rapid palatal expansion: a prospective, randomized clinical trial. Am J Orthod Dentofacial Orthop 2005; 128:299-309.

[61] Vesse M. Classe III squelettiques. Encycl Méd Chir, Paris : Elsevier;1999, 23-472-G-10:1-18.

[62] Vesse M. Traitement précoce des classes III : justification théoriques et cliniques. Rev Orthop Dento Faciale 2003;37:305-328

[63] Westwood PV, McNamara JA Jr, Baccetti T, Franchi L, Sarver DM. Long term effects of Class III treatment with rapid maxillary expansion and facemask therapy followed by fixed appliances. Am J Orthod Dentofacial Orthop 2003; 123:306-320 